\title{
A case of mistaken identity: alcohol withdrawal, schizophrenia, or central pontine myelinolysis?
}

\author{
This article was published in the following Dove Press journal: \\ Neuropsychiatric Disease and Treatment \\ 23 January 2012 \\ Number of times this article has been viewed
}

\author{
Paul Schneider \\ Vicki A Nejtek ${ }^{2,3}$ \\ Cheryl L Hurd ${ }^{2,3}$ \\ 'Green Oaks Behavioral Health Care \\ Services, Dallas, ${ }^{2}$ University of North \\ Texas Health Science Center, Fort \\ Worth, ${ }^{3}$ John Peter Smith Health \\ Network, Fort Worth, Texas, USA
}

\begin{abstract}
Demyelination is a hallmark of central pontine myelinolysis (CPM). Neuropsychiatric manifestations of this condition include weakness, quadriplegia, pseudobulbar palsy, mood changes, psychosis, and cognitive disturbances. These psychiatric symptoms are also associated with schizophrenia and alcohol withdrawal. Thus, it is clinically relevant to differentiate between CPM, schizophrenia, and alcohol withdrawal as the treatment and prognostic outcomes for each diagnosis are distinct. We present a series of events that led to a misdiagnosis of a patient admitted to the medical emergency center presenting with confusion, psychomotor agitation, and delirium who was first diagnosed with schizophrenia and alcohol withdrawal by emergency medical physicians and later discovered by the psychiatric consult team to have CPM. With a thorough psychiatric evaluation, a review of the laboratory results first showing mild hyponatremia (127 mmol/L), subsequent hypernatremia $(154 \mathrm{mmol} / \mathrm{L})$, and magnetic resonance brain imaging, psychiatrists concluded that CPM was the primary diagnosis underlying the observed neuropsychopathology. This patient has mild impairments in mood, cognition, and motor skills that remain 12 months after her emergency-center admission. This case report reminds emergency clinicians that abnormal sodium metabolism can have long-term and devastating psychopathological and neurological consequences. Differentiating between CPM, schizophrenia, and alcohol withdrawal using neuroimaging techniques and preventing the risks for CPM using slow sodium correction are paramount.
\end{abstract}

Keywords: MRI, alcohol, schizophrenia, central pontine myelinolysis, hyponatremia

\section{Background}

Central pontine myelinolysis (CPM) is a rare neurologic disorder of demyelination that was first reported by Adams et al over 50 years ago in a malnourished 38-year-old man with alcohol dependence. ${ }^{1}$ In addition to malnourishment and alcohol dependence, the Adams et al case-report patient was also described as having hyponatremia. Since that time, several studies have shown that rapid overcorrection of hyponatremia exacerbates CPM as well..$^{2-11}$ Common symptoms of CPM include weakness, quadriplegia, pseudobulbar palsy, behavioral changes, psychosis, and cognitive disturbances. ${ }^{1-13}$ Behavioral changes, psychosis, and some cognitive disturbances are psychiatric symptoms that meet Diagnostic and Statistical Manual of Mental Disorders, Fourth Edition (DSM-IV) ${ }^{14}$ criteria for schizophrenia ${ }^{15}$ and are often observed in alcohol delirium psychosis, ${ }^{13,16-18}$ and hyponatremia. ${ }^{3,13,19}$

The mortality rate in CPM patients is reportedly between 5\%-10\% and the prognosis is generally considered poor. ${ }^{3,5-8,13,20}$ Thus, it is critically important to recognize CPM in an emergency room setting. However, given the rarity of CPM, it is understandable how 
a patient exhibiting psychogenic symptoms may appear to an emergency-room physician as a case of alcohol withdrawal delirium or schizophrenia rather than CPM. As the treatment for CPM, schizophrenia, and alcohol delirium are distinct, it is clinically relevant to differentiate between these diagnoses and determine the presence of other possible medical or psychiatric disorders.

To our knowledge, this is one of the few retrospective case reports with detailed reports and 1-year follow-up documentation to describe behavioral symptoms, laboratory results, treatment outcomes, and disposition of a CPM patient who was initially misdiagnosed with schizophrenia. Magnetic resonance images showing brain morphology documented in this case are also provided. The main goals of this case report are to help emergency clinicians understand the importance of ruling out neurological disorders prior to diagnosing a patient with a serious mental illness, assist them in recognizing that psychogenic behaviors may be associated with CPM and sodium overcorrection, aid physicians in recognizing the value of requesting a psychiatric consult early in cases where patients exhibit any psychogenic symptoms, and to help emphasize the importance of using magnetic resonance imaging (MRI) brain scans to facilitate accurate diagnoses.

\section{Protection of human subjects}

The Institutional Review Board from the John Peter Smith Health Network and the University of North Texas Health Science Center provided approval to conduct a retrospective chart review. The medical chart review conducted in December 2010 describes a patient who was provided services in the JPS Health Network between December 2009 and 2010.

\section{Case report}

A single, 30-year-old African American female living on the streets and drinking alcohol heavily was found partially nude, aggressive, and talking to people who were not present. She was brought to the psychiatric emergency center by the police. The patient's body mass index (21.79) indicated that she was not underweight. There was no evidence of seizure activity or head injury, and there were no previous hospitalizations for mental illness, drug or alcohol problems, no self-injurious or suicidal behaviors. On admission, the patient was not using any medications and had no allergies. During the initial psychiatric emergency center intake, a noticeable tremor was observed and thus, the patient was immediately transferred to the medical emergency center for further evaluation.
Upon arrival at the emergency center, the patient developed respiratory arrest and was intubated in the intensive care unit. The patient's albumin level was $2.7 \mathrm{~g} / \mathrm{dL}$ (normal range $=3.4-5.0 \mathrm{~g} / \mathrm{dL}$ ). Laboratory results in the emergency center showed that the patient had a serum sodium level of $127 \mathrm{mmol} / \mathrm{L}$ which was $6 \mathrm{mmol} / \mathrm{L}$ lower than the normal range of $133-145 \mathrm{mmol} / \mathrm{L}$. Serum creatinine was $1.6 \mathrm{mg} / \mathrm{dL}$ (normal range is $0.5-1.4$ ), potassium was $3.4 \mathrm{mEq} / \mathrm{L}$ (normal range is $3.5-5.3)$, and serum ethanol was $<10 \mathrm{mg} / \mathrm{dL}(0.01$ blood alcohol). Blood urea nitrogen was $46 \mathrm{mg} / \mathrm{dL}$ (normal 5-25). The total white blood cell count was $18.6 \times 10^{3} / \mathrm{cmm}$ (normally $4.50-11.00 \times 10^{3} / \mathrm{cmm}$ ), $82 \%$ were neutrophils, hemoglobin concentration was $7.5 \mathrm{~g} / \mathrm{dL}$ (normally $12.0-16.0 \mathrm{~g} / \mathrm{dL}$ ), and hematocrit was $23.7 \%$ (normally $36.0 \%-46.0 \%$ ).

Urinalysis was positive for leukocyte esterase, bacteria, 281 white blood cells, and E. coli, and was negative for any substance use. Urine osmolarity was $324 \mathrm{mOsm} / \mathrm{kg}$, chloride was $102 \mathrm{mEq} / \mathrm{L}$, and sodium was $99 \mathrm{mEq} / \mathrm{L}$. There was no evidence of polydipsia. The electrocardiogram indicated that the heart rate was 80 (normal range of 60-100 beats per minute), the pulse rate was 155 , QT/QTc were $384 / 419 \mathrm{~ms}$, the QRS was $9 \mathrm{~ms}$, the QRSD was $93 \mathrm{~ms}$, and T wave was $-5 \mathrm{~ms}$. There was no evidence of bradycardia or tachycardia, and normal ventricular depolarization and repolarization were reported.

The patient was admitted as an inpatient with the following medical diagnoses: (1) metabolic encephalopathy due to urosepsis or delirium, (2) hyponatremia, (3) hypokalemia, (4) acute renal failure, and (5) anemia. Although serum alcohol and urine drug screen analyses were negative, she was given intravenous (IV) thiamine, folate, and oral chlordiazepoxide $50 \mathrm{mg}$ three times a day for possible alcohol withdrawal. She was started on IV fluids (D5 normal saline at $120 \mathrm{cc} /$ hour) for the acute renal failure, and hypokalemia was treated with $40 \mathrm{mEq} / \mathrm{L}$ of potassium chloride. The urinary tract infection was treated with IV ciprofloxacin.

On hospital day 2, hemoglobin and hematocrit were $6.6 \mathrm{~g} / \mathrm{dL}$ and $21.6 \%$, respectively. Within 18 hours of transfusion of two units of packed red blood cells and an antibiotic change to piperacillin/tazobactam, the acute renal failure resolved. The emergency medical team elevated serum sodium at $8-10 \mathrm{mmol} / \mathrm{L}$ as per recommended guidelines $^{7-11}$ for acute mild hyponatremia (127 mmol/L). However, the patient's serum sodium rose from $127 \mathrm{mmol} / \mathrm{L}$ to $141 \mathrm{mmol} / \mathrm{L}$, approximately 17 hours after the patient was first admitted to the medical emergency center. 
On day 4, serum sodium levels rose from $141 \mathrm{mmol} / \mathrm{L}$ to $156 \mathrm{mmol} / \mathrm{L}$ resulting in hypernatremia. No chart information was available to explain the cause of the sodium fluctuation, especially since the patient originally had mild hyponatremia and the recommended sodium level guidelines were followed.

On day 4, a psychiatry consult was ordered to evaluate the patient for confusion, psychomotor agitation, and oppositional behavior. During the consult, the progress notes show that the patient believed that the reason for her hospitalization was "because one day my boyfriend ticked me off." She refused to speak unless given a drink of water and she reported seeing people on the other side of the room with whom she began conversing. She was verbally agitated and oppositional, and threatened to harm someone if she did not get a drink of water. She was lethargic with shifting sensorium and fluctuation of attention and orientation occurred during the psychiatric interview.

As the patient had no known history of mental illness, the psychiatric consult team suspected CPM due to elevated serum sodium levels. The psychiatric team ordered an MRI scan, an electroencephalogram and a computed tomography (CT) brain scan to assist them in making an accurate diagnosis. In addition, they treated the patient with lorazepam to alleviate her agitation and non-specific delirium until the presence of severe mental illness could be determined.

The CT brain scan was negative and showed no evidence of any acute hemorrhage, infarct, lesion, or mass. However, the MRI scan showed a pontine lesion and hyperintensity in lenticular nuclei. The lenticular nuclei consist of the caudate nucleus, putamen, and the globus pallidus, and pathological behaviors associated with lenticular nuclei hyperintensities include dystonia and sometimes abulia. ${ }^{13}$ The electroencephalogram showed a normal awake and drowsy pattern with anterior beta and theta frequency rhythm and low voltage alpha activity $(8.5$ to $9 \mathrm{~Hz})$ in the posterior occipitotemporal region.

After psychiatrists discovered the central pontine lesion with MRI on day 4, they determined that the observed pathological behaviors were the result of CPM and sodium overcorrection. However, on day 7, emergency physicians diagnosed the patient with catatonic schizophrenia and alcohol withdrawal delirium, and ordered a second psychiatric consult. On day 8, the patient was lethargic, but was not paralyzed or frozen; eyes were open and blinking; mouth drooling, lips appeared to slightly move as if attempting to communicate, but SHE remained non-verbal.
She responded only to noxious stimuli; she was cognizant enough to squeeze the examiner's finger with prompting and followed appropriate eye-tracking.

The second psychiatric consult concluded as in the first consult that there was no evidence to support a DSM-IV diagnosis for catatonic schizophrenia. In contrast to emergency physicians' diagnoses, psychiatrists once again documented that the blood alcohol level of $<0.01$ did not support a diagnosis of alcohol withdrawal delirium. On days 6 and 7, the patient's serum sodium levels reached a maximum of $159 \mathrm{mmol} / \mathrm{L}$ which later decreased to $152 \mathrm{mmol} / \mathrm{L}$ on day 8 . In line with the literature, ${ }^{3-11,19}$ the overcorrection of sodium in this patient was the most important clue that should have raised in emergency physicians the suspicion of central pontine myelinolysis rather than severe mental illness. The diagnosis disconnect between emergency physicians and the psychiatric consult team is concerning.

\section{Inpatient neuroimaging}

At week 5, the psychiatric consult team ordered a second MRI scan and the results showed a $2-3 \mathrm{~cm}$ lesion in the central pons. The pontine lesion and lenticular abnormalities identified in the first MRI scan were still evident in the second. A third MRI scan taken at week 8 again showed the same brain morphology seen in the two previous images. The MRI signals for all three scans were non-enhancing and suggestive of chronic osmotic demyelination. Similar to other studies, ${ }^{21}$ all three MRI brain scans showed an abnormal illdefined increased signal in the central pons most consistent with CPM (Figure 1).

Atrophy in the prefrontal cortex, temporal cortex, hippocampi, amygdala, corpus callosum, and thalamus is common in schizophrenia. ${ }^{15}$ Thalamic dysfunctioning and cerebellar atrophy have been identified as a primary brain abnormality in alcohol psychosis. ${ }^{17-22}$ In line with the literature, none of the neuroimages supported a schizophrenia diagnosis or an alcohol use disorder.

\section{Prognostic outcome}

After 2 months of inpatient recovery, this 30-year-old patient was discharged to a nursing home. She was started on citalopram $20 \mathrm{mg}$ daily for depression. After 2 weeks of skilled nursing-home care, she returned to the hospital after again becoming confused, showing psychomotor agitation and visual hallucinations similar to previous accounts. One milligram of risperidone two times a day was prescribed as an adjunctive medication to citalopram, and within a few 

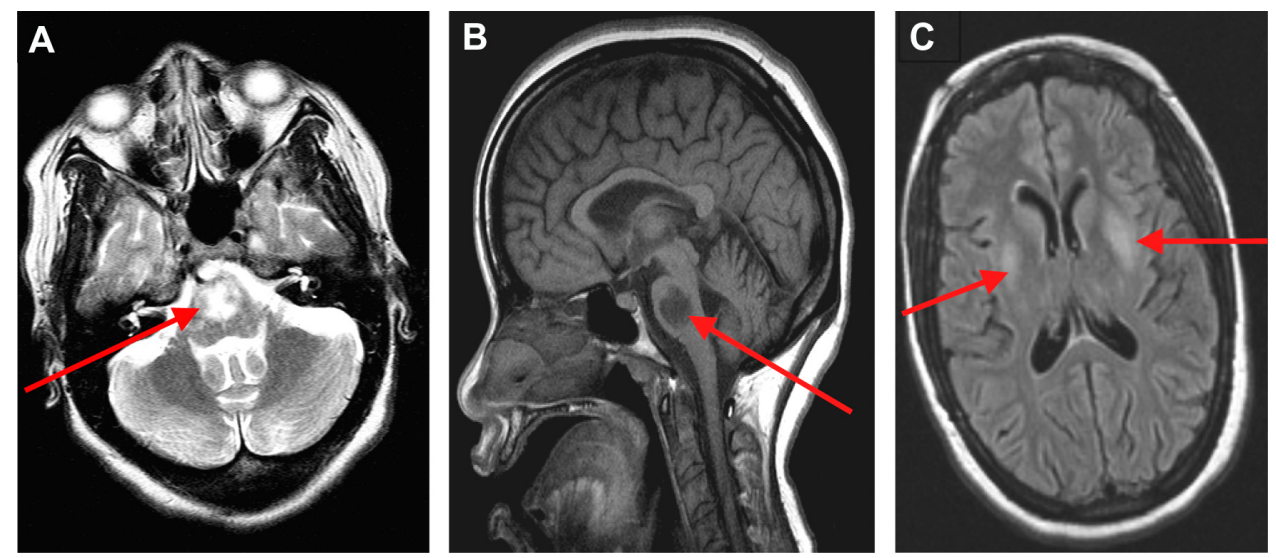

Figure I TI- and T2-weighted MRI data was acquired with the GE I.5-Tesla magnetic resonance scanner (General Electric Healthcare Technologies, Waukesha, WI). A pontine lesion in axial (A) and sagittal (B) views, and lentiform hyperintensity in axial plane (C) are shown.

hours she was transferred to the hospital's skilled nursing unit to receive physical therapy. When she began to ambulate independently with a rolling walker and could perform all activities of daily living, she was discharged to her home.

Following discharge to her home, the patient received once monthly follow-up for 1 year with the outpatient psychiatry clinic team. The last documented pharmacotherapy regimen included $20 \mathrm{mg}$ daily of citalopram, $1 \mathrm{mg}$ of risperidone, and $300 \mathrm{mg}$ of gabapentin twice daily. Over time, she gained good insight and self-described her psychiatric condition as a result of "brain damage." Her final psychiatric diagnosis was documented as a psychotic disorder secondary to a general medical condition (ie, CPM). Although this patient experienced substantial recovery of functioning since her emergency hospital admission 12 months earlier, mild impairments in mood, cognition, and motor skills requiring the use of a walker remain.

\section{Conclusion}

Many chronic and acute diseases present with symptoms that may overlap with common psychiatric syndromes. CPM is one of many medical syndromes that may present with psychosis during the course of the illness. The most obvious symptoms in this case report were a frank loss of attention, apraxia, increased irritability, and psychomotor agitation. Labile mood and cognitive changes in this patient included disorientation, confusion, threatening angry outbursts, flat affect, loose associations, and lethargy. The emergency medical team considered these cluster symptoms as primary factors in diagnosing the patient with catatonic schizophrenia rather than sodium fluctuations.

It is important for emergency personnel to understand that catatonia is a rare syndrome characterized by lack of interaction with the environment and is accompanied with specific motor symptoms, including bizarre posturing and maintenance of awkward postures into which the patient is placed (waxy flexibility). ${ }^{15}$ In addition, persons affected with catatonia offer purposeless resistance to commands with repetitive and meaningless gestures and activities. ${ }^{15}$ Based on the mix of behaviors observed in this patient, the lack of waxy flexibility and her abilities to respond to noxious stimuli and follow appropriate eye-tracking movements indicate that the DSM-IV criteria for catatonia ${ }^{14}$ were completely absent. Moreover, none of the patient's laboratory results supported alcohol withdrawal.

Neuroimaging is not routinely utilized as a psychiatric diagnostic tool in the emergency care setting. However in this particular case, neuroimaging results were critically important for the differential diagnosis and the recognition that this patient's symptoms were most likely the result of CPM. As this patient did not have a history of any DSM-IV Axis I disorder, a neurological consult and neuropsychological testing would have also been very helpful. In the process of elimination and the diagnoses offered by the psychiatry consult team, the continued focus on severe mental illness and substance abuse by the emergency medicine team is perplexing.

The severe shifts in sodium levels such as seen in this patient are well-known causes of psychopathological behaviors that are best resolved with slow and steady sodium correction..$^{3-11,23}$ In speculative hindsight, the severe sodium fluctuations may have been due to inappropriate antidiuretic hormone release. According to data presented by Rose and Post, ${ }^{24}$ Steele et al, ${ }^{25}$ and Adler et $a l,{ }^{26}$ the isotonic saline the patient received (120 cc/hour) paired with $40 \mathrm{mEq} / \mathrm{L}$ of potassium chloride and paired with the IV ciprofloxin to treat 
the urinary tract infection may have induced an inappropriate antidiuretic hormone release. In the absence of polydipsia, water or alcohol intoxication, either inappropriate antidiuretic hormone release or spontaneous overcorrection of sodium are the only two logical explanations available.

This case illustrates the need for early MRI studies when sodium fluctuations are present and no psychiatric illness or substance abuse histories are noted. The series of events presented here highlight the need for emergency physicians to consider psychopathological behaviors as a sequelae of acute urosepsis and sodium fluctuation from hyponatremia to hypernatremia. ${ }^{23}$ Importantly, slowly correcting sodium levels in patients showing behavioral abnormalities cannot be overemphasized as the mortality rate is $50 \%$ or greater in those with hypernatremia. ${ }^{23}$

Emergency medical professionals must recognize the need for an immediate psychiatric consult to obtain a thorough psychiatric history before diagnosing a patient with a serious mental illness. Otherwise, important clinical features may be overlooked such as in this patient's case. A psychiatric consult before day 4 may have facilitated this patient's recovery and prognosis. It is imperative to rule out metabolic and neurological abnormalities with appropriate neuroimaging and laboratory tests prior to diagnosing a patient with a severe psychiatric diagnosis such as catatonic schizophrenia or labeling patients with alcohol withdrawal.

With regard to long-term care, once the patient left the purview of the psychiatric consult team and was transitioned to outpatient services, no neuroimaging tests or neuropsychological evaluations were ordered during the 12-month follow-up period. The lack of both neuroimaging and neuropsychological tests to determine cognitive prognostic outcomes is concerning. A chart review suggested that failure to provide these tests may reflect the patient's insurance benefit restrictions. Nevertheless, an obvious limitation found in the follow-up outpatient care of this patient is that without the use of objective testing tools, the neurological long-term prognosis in this patient remains uncertain.

\section{Acknowledgments}

Drs Schneider, Nejtek, and Hurd reviewed the medical chart, assimilated the clinical data, provided data interpretation, and scientific writing for publication. As Dr Nejtek has expertise in psychiatry neuroimaging, she also chose the images to present in the case study. All authors conducted literature searches for this case study and provided data interpretations and edits that shaped the final manuscript.
Presented, in part, at the American Academy of Addiction Psychiatry 21st Annual Meeting and Symposium held December 2-5, 2010 in Boca Raton, Florida. This study was conducted without external funding.

\section{Disclosure}

The authors have no financial or nonfinancial competing interests related to this case report.

\section{References}

1. Adams RD, Victor M, Mancall EL. Central pontine myelinolysis: a hitherto undescribed disease occurring in alcoholic and malnourished patients. AMA Arch Neurol Psychiatry. 1959;81(2):154-172.

2. Aleu FP, Terry RD. Central pontine myelinolysis. Arch Pathol. 1963;76: 140-146.

3. Bolton CF, Young GB, Zochodne DW. The neurological complications of sepsis. Ann Neurol. 1993;33:94-100.

4. Brunner JE, Redmond JM, Haggar AM, Elias SB. Central pontine myelinolysis after rapid correction of hyponatremia: a magnetic resonance imaging study. Ann Neurol. 1988;23(4):389-391.

5. Chen R, Young GB. Metabolic encephalopathies. In: Bolton CF, Young GB, editors, Ballière's Clinical Neurology. London: Ballière Tindall, 1996:577.

6. Karp BI, Laureno R. Pontine and extrapontine myelinolysis: A neurologic disorder following rapid correction of hyponatremia. Medicine (Baltimore). 1993;72(6):359-373.

7. Laureno R, Karp BI. Myelinolysis after correction of hyponatremia. Ann Intern Med. 1997;126(1):57-62.

8. Martin RJ. Central pontine and extrapontine myelinolysis: The osmotic demyelination syndromes. J Neurol Neurosurg Psychiatry. 2004;75(Suppl 3):22-28.

9. Nielsen JM. Central pontine myelinolysis complicating hyponatremia Med J Aust. 1987;146:492-494.

10. Norenberg MD, Leslie KO, Robertson AS. Association between rise in serum sodium and central pontine myelinolysis. Ann Neurol. 1982;11:128-135.

11. Peces R, Ablanedo P, Alvarez J. Central pontine and extrapontine myelinolysis following correction of severe hyponatremia. Nephron. 1988;49:160-163.

12. Hagiwara K, Okada Y, Shida N, Yamashita Y. Extensive central and extrapontine myelinolysis in a case of chronic alcoholism without hyponatremia: A case report with analysis of serial MR findings. Inter Med. 2008;47:431-435. [Epub March 3, 2008.]

13. Bhatia KP, Marsden CD. The behavioural and motor consequences of focal lesions of the basal ganglia in man. Brain. 1994;117(Pt 4): 859-876.

14. Nasrallah HA. Neurologic comorbidities in schizophrenia. J Clin Psychiatry. 2005;66(Suppl 6):34-46.

15. First M, Spitzer R, Gibbon M, Williams J. Structured Clinical Interview for DSM-IV Axis I Disorders - Clinical Version (SCID-CV). Washington DC: American Psychiatric Press; 1996.

16. McNichol RW. The Treatment of Delirium Tremens and Related States Springfield, IL: Thomas; 1970:708-712.

17. Chamess ME, Simon RP. Greenberg DA: Ethanol and the nervous system. N Engl J Med. 1989;321:442-445.

18. KitabayashiY, Narumoto J, Shibata K, Ueda H, Fukui K. Neuropsychiatric background of alcohol hallucinosis: A SPECT study. J Neuropsychiatry Clin Neurosci. 2007;19:1,85.

19. Singh RK, Chaudhury S. Hyponatremia-induced psychosis in an industrial setting. Ind Psychiatry J. 2009;18:137-138.

20. Yoon B, Shim YS, Chung SW. Central pontine and extrapontine myelinolysis after alcohol withdrawal. Alcohol Alcohol. 2008;43(6): 647-649. 
21. DeWitt LD, Buonanno FS, Kistler JP, et al. Central pontine myelinolysis: demonstration by nuclear magnetic resonance. Neurology. 1984;34(5): 570-576.

22. Soyka M, Zetzsche T, Dresel S, Tatsch K. FDG-PET and IBZM-SPECT suggest reduced thalamic activity but no dopaminergic dysfunction in chronic alcohol hallucinosis. J Neuropsychiatry Clin Neurosci. 2000;12: 287-288.

23. Mandal AK, Saklayen MG, Hillman NM, Markert RJ. Predictive factors for high mortality in hypernatremic patients. Am J Emerg Med. 1997; 15(2):130-132.
24. Rose BD, Post TW. Clinical Physiology of Acid-Base and Electrolyte Disorders, 5th ed. New York: McGraw-Hill; 2001:703-711.

25. Steele A, Gowrishankar M, Abrahamson S, Mazer CD, Feldman RD, Halperin ML. Postoperative hyponatremia despite nearisotonic saline infusion: a phenomenon of desalination. Ann Intern Med. 1997;126(1):20-25.

26. Adler D, Voide C, Thorens JB, Desmeules J. SIADH consecutive to ciprofloxacin intake. Eur J Intern Med. 2004;15(7):463-464.

\section{Publish your work in this journal}

Neuropsychiatric Disease and Treatment is an international, peerreviewed journal of clinical therapeutics and pharmacology focusing on concise rapid reporting of clinical or pre-clinical studies on a range of neuropsychiatric and neurological disorders. This journal is indexed on PubMed Central, the 'PsycINFO' database and CAS.
The manuscript management system is completely online and includes a very quick and fair peer-review system, which is all easy to use. Visit http://www.dovepress.com/testimonials.php to read real quotes from published authors.

Submit your manuscript here: http://www.dovepress.com/neuropsychiatric-disease-and-treatment-journal 